\title{
Enhance Thermoelectric and Mechanical Properties of Thermo- plastic Vulcanizates (TPVs) by Constructing Nanosheet Network of Hydroxylated Graphene
}

\author{
Qi Tang ${ }^{1,2}$, Lan Cao ${ }^{1}$, Xiurui Lang ${ }^{2}$, Yingxia Zong ${ }^{3}$ and Chengzhong Zong ${ }^{1,2, *}$ \\ 1 School of Materials Science and Engineering, University of Science and Technology Qingdao, Qingdao \\ 266042, China; Tangqi@qust.edu.cn (Q.T.); 15953228331@163.com (X.L.) \\ 2 Key Laboratory of Rubber-plastics of Ministry of Education, Qingdao 266042, China; 13646397867@163.com \\ (L.C.) \\ College of Chemical and Engineering, University of Science and Technology Qingdao, Qingdao 266042, \\ China; 15063946968@163.com (Y.Z.) \\ * Correspondence: polymer@qust.edu.cn (C.Z.); Tel.: +86-130-0653-9866
}

\begin{abstract}
In order to obtain higher thermoelectric and mechanical properties in non-polar thermoplastic vulcanizates (TPVs), the Butyl rubber/Polypropylene (TPVs)/hydroxylated graphene (HGE) composites with nanosheet network were prepared through masterbatch technique and based on thermodynamic calculations, using polypropylene-graft-maleic anhydride (PP-MA) as a compatibilizer. The FTIR and Raman spectra revealed the introduced maleic anhydride group on PP-MA can form strong interfacial interaction with hydroxyl-containing functional groups on HGE. Morphology study indicated the rubber particles in the composites occupied the most volume of the PP phase, as expected to hinder the aggregation of HGE and form the effective nanosheet network. The nanosheet network can be combined with the IIR cross-linked particles during the dynamic vulcanization process to improve the interface bonding between PP and IIR, thus increasing the tensile strength of TPVs. When the content of HGE reached the percolation threshold (2 wt.\%), the nanosheet network of HGE was formed, and the AC conductivity, dielectric permittivity and thermal conductivity increased sharply. The prepared TPVs/HGE nanocomposites have significantly improved in mechanical properties, thermal properties and dielectric properties, which provides a guarantee for their potential application as multifunctional TPVs polymers.
\end{abstract}

Keywords: thermoplastic vulcanizates; graphene; butyl rubber; polypropylene

\section{Introduction}

Thermoplastic vulcanizates (TPVs) is a special polymer material that has the elasticity of cured rubber at room temperature and can be repeatedly processed as thermoplastic [1]. In recent years the addition of nanofillers into TPVs has been commonly used for preparing high-performance conductive elastomers [2-Error! Reference source not found.]. Compared with conventional rigid conductive polymer materials, conductive TPVs composites with good flexibility and considerable elasticity hold great potential for conductors, strain sensors, antistatic coatings and actuators. The traditional method to increase the electrical properties of TPVs was to add carbon black or metal powder to the matrix, but the typical loading is usually higher than $50 \%$, resulting in poor mechanical properties and processing properties [4-6]. Recently, graphene-based polymer composites have attracted considerable attention from both fundamental research and application prospects of view due to excellent electrical properties and low-conductive percolation threshold [Error! Reference source not found.-8].

Graphene with unique sp²-hybridized carbon atomic structure and two-dimensional layered carbon nanomaterials have attracted numerous attentions in the recent years for its unique combination of high mechanical strength, electrical conductivity, and thermal 
conductivity [9]. Compared with conventional nanofillers, it holds great promise for potential applications in the fields of energy storage systems, biomedical science and highperformance composites [10-11]. Many studies have focused on improving polymer properties by adding graphene to prepare advanced multifunctional composites [12-13]. However, the main challenges on the preparing of high-performance composites/graphene is the inert surface structure of graphene and the weak interaction between the two components, so the wetting behavior and interfacial bonding are vital to achieve excellent properties. Yan et al. [Error! Reference source not found.] used graphene oxide as compatibilizer to prepared natural rubber (NR)/high density polyethylene (HDPE) thermoplastic vulcanizates by an ultrasonically assisted latex mixing process (ULM). Graphene helped to enhance the interfacial adhesion and phase compatibility, and the tensile strength and tensile modulus showed significant enhancement in the composites. However, chemical modification methods had complex processes and may destroy the atomic structure of the nanofillers, which would affect the conductivity of the composites [14]. So far, few investigations were reported concerning the comprehensive properties of TPVs/graphene composites especially for the thermoelectric and mechanical properties.

Since TPVs has special phase structure (high content of cross-linked rubber as the dispersed phase and low content of thermoplastic as the continuous phase), we hope to build nanosheet network by uniformly dispersing HGE in a low-content thermoplastic resin. In this work, inspired by the unique network of TPVs, we prepared the TPVs/HGE composites with nanosheet network through masterbatch technique and based on thermodynamic calculations, using PP-MA as a compatibilizer. The introduced maleic anhydride group on PP-MA is expected to create strong interfacial interaction with hydroxylcontaining functional groups on HGE, thus leading to the efficient dispersion of HGE during melt processing with PP. The rubber particles in the composites occupies the most volume of PP phase, as expected to hinder the aggregation of HGE and form the effective nanosheet network. The nanosheet network in the composites can be formed by overlapping graphene evenly dispersed in PP, which can improve the AC conductivity and thermal conductivity. More importantly, the nanosheet network could improve the interface bonding of PP and IIR and improve the elasticity of the TPV matrix. The prepared composites show significant enhancements in thermal, electrical and mechanical properties, ensuring its potential applications functional TPVs composites.

\section{Materials and Methods}

\subsection{Materials}

The hydroxylated graphene sheets (HGE) with a hydroxyl content of $7.8 \%$ and BET surface area of $400-600 \mathrm{~m}^{2} / \mathrm{g}$ were purchased from Shandong Shengquan New Material Co., Ltd., China. PP PP4220 (melt flow index: $0.36 \mathrm{~g} / 10 \mathrm{~min}$ at $210^{\circ} \mathrm{C}$ and $2.16 \mathrm{~kg}$, p=0.89 $\mathrm{g} / \mathrm{cm}^{3}$ ) were purchased from Sinopec Yanshan Petrochemical Company, China. A commercially available polypropylene-graft-maleic anhydride (PP-MA) were provided by Arkema, France. The IIR material was Butyl 1675 from LANXESS, with an unsaturation of $1.85 \mathrm{~mol} \%$. Pentaerythritol tetrakis 3-(3,5-di-tert-butyl-hydroxyphenyl) propionate (Irganox 1010) was used as an antioxidant and was provided by Shanghai Shanpu Chemical Co., Ltd., China. P-(1,1,3,3-tetramethylbutyl)- phenol (RT4201) was used as curing agents provided by Shengquan industry company, China. Stannous chloride, dihydrate $\left(\mathrm{SnCl}_{2} \cdot 2 \mathrm{H}_{2} \mathrm{O}\right)$ (purity $>98 \%$ ) was obtained from Tianjin Beilian Fine Chemical Development Co., Ltd., China.

\subsection{Preparation of TPVS/HGE composites}

\subsubsection{Preparation of PP-MA/HGE masterbatch}

PP-MA/HGE masterbatch was prepared using PP-MA as a compatibilizer in a Hakke intermal mixer (Harbin Hapu Electric Technology Co., Ltd, RM-200C, China) equipped 
with roller rotors. PP-MA and PP were dried in a vacuum oven at $100{ }^{\circ} \mathrm{C}$ for about 12 hours before being used. PP-MA, HGE and 1010 (1.0 wt.\% based on PP-MA) were added to the RM-200C with a rotor speed of $60 \mathrm{rpm}$ at $180{ }^{\circ} \mathrm{C}$, and melt-mixed for 5 min to prepare PP-MA/HGE masterbatch. In this work, the weight ratio of PP-MA versus HGE was $4: 1$.

\subsubsection{Preparation of TPVs/HGE composites}

The prepared PP-MA/HGE masterbatch was crushed using a pestle and then melt compounded with pure PP and IIR to obtain compatibilized homogeneous blends. The weight ratio of IIR versus PP was 6:4. Subsequently, the blends was transferred to another two-roll mill (Baolun Precision Testing Instrument Co., Ltd, BL-6175-BL, China) at ambient temperature, and RT4201 (10.0 wt.\% based on IIR rubber) and $\mathrm{SnCl}_{2} \cdot 2 \mathrm{H}_{2} \mathrm{O}(2.0 \mathrm{wt}$.\% based on RT4201) were added to obtain undynamically vulcanized composites. Finally, the premix was fed into the RM-200C at $180{ }^{\circ} \mathrm{C}$ and a rotor speed of $120 \mathrm{rpm}$, and TPVs/HGE composites were obtained after dynamic vulcanization for $6 \mathrm{~min}$. The obtained samples were compression molded at $185^{\circ} \mathrm{C}$ for $10 \mathrm{~min}$.

\subsection{Characterization}

The interfacial interaction between HGE and PP-MA was characterized by a FTIR spectrometer (Bruker Vertex 70, Germany) with the scan range of $400-4000 \mathrm{~cm}^{-1}$ in ATR mode. The interfacial interaction was also characterized by a Renishaw Invia Reflex Raman system (Renishaw 1000, Germany) with the $532 \mathrm{~nm}$ laser was used as the light source and the excitation light source is He-Ne laser. Surface energy and interfacial tension were calculated by measuring the contact angles by using a digital automated contact angle goniometer (KRUSS, DSA100, Germany) at $25{ }^{\circ} \mathrm{C}$. Double distilled $\left(\mathrm{H}_{2} \mathrm{O}\right)$ and glycerin $\left(\mathrm{C}_{3} \mathrm{H}_{8} \mathrm{O}_{3}\right)$ were used as probe liquids. The contact angles of all samples were carried out for at least 5 times. The morphology study of the TPVs/HGE composites was tested using TEM (JEOL, JEM-1200EX, Japan) at an accelerating voltage of $200 \mathrm{KV}$. The samples were cryomicrotomed into sections with a thickness of $50 \mathrm{~nm}$ at $-100{ }^{\circ} \mathrm{C}$ by using a microtome (LEICA, Germany) before TEM observation. Tensile properties of the TPVs/HGE composites were measured as per ASTM D 412 by using a universal testing machine (Zwick Z005, Germany) at room temperature with the speed of $500 \mathrm{~mm} / \mathrm{min}$. The values were calculated as averages over five specimens for each composition. The tensile fracture morphology of the pure TPVs and TPVs/HGE composites was investigated by using SEM (JEOL, JEM-2100F, Japan) operating at $20 \mathrm{KV}$. Before characterization, the samples coated with gold. The dielectric properties were measured by using a broadband dielectric spectroscopy (Novocontrol GmbH, Concept 80, Germany) at room temperature over the frequency range from $10^{\circ} \mathrm{Hz}$ to $10^{6} \mathrm{~Hz}$. The samples were prepared as sheets with a diameter of about $20 \mathrm{~mm}$ and a thickness of about $2 \mathrm{~mm}$. Both flat sample surfaces were painted with a layer of high-purity gold paint to from electrodes. The thermal conductivity of each sample was measured by thermal conductivity instrument (DTC-300, TA, USA) according to ASTM D5470.

\section{Results and Discussions}

\subsection{PP-MA/HGE masterbatch analysis}

Currently, the uniform dispersion of graphene is essential for the preparation of highperformance TPVs composites, mainly for those needing high electrical conductivity. In this work, we expect to achieve uniform dispersion of HGE through masterbatch technique, using PP-MA as a compatibilizer. The introduced maleic anhydride group on PP$\mathrm{MA}$ is expected to create strong interfacial interaction with hydroxy-containing functional groups on HGE, thus leading to the efficient dispersion of HGE during melt processing with PP. To study the interfacial interaction between HGE and PP-MA, Figure 1(A) shown 
the FTIR spectra of pure PP, PP-MA/HGE masterbatch and TPVs/HGE composite containing 2 wt.\% HGE. The characteristic bands at $1778 \mathrm{~cm}^{-1}$ and $1717 \mathrm{~cm}^{-1}$ represent the $\mathrm{C}=\mathrm{O}$ stretching vibration of maleic anhydride of PP-MA [15]. Besides, the strong peak observed between $1000 \mathrm{~cm}^{-1}$ and $1200 \mathrm{~cm}^{-1}$ represents the $\mathrm{C}-\mathrm{O}$ stretching vibration. For the PPMA/HGE masterbatch and TPVs/HGE composite, the characteristic peaks at $1778 \mathrm{~cm}^{-1}$ and $1717 \mathrm{~cm}^{-1}$ become weak, indicating the form of large numbers of hydrogen bonds on the maleic anhydride group with HGE, which would lead to the significant decrease in peak intensity or the disappearance of maleic anhydride groups [16].

Raman spectra can further characterize the interfacial interaction between HGE and PP-MA. Raman spectra of HGE, PP-MA/HGE masterbatch and TPVs/HGE composites (2 wt.\% HGE) were carried out, the results were shown in Figure 1(B). In the Raman spectra of HGE, one can observe the prominent peaks at $1586 \mathrm{~cm}^{-1}$ and $1348 \mathrm{~cm}^{-1}$, which belong to the $D$ band and $G$ band of graphene [17]. For PP-MA/HGE masterbatch and TPVs/HGE composites, the $\mathrm{G}$ band is blue shift about $7 \mathrm{~cm}^{-1}$ comparing with neat HGE. The blue shift of the $\mathrm{D}$ band indicates that there is a strong interface interaction between the remaining functional groups on HGE and PP-MA [18]. The intensity ratio ( $\left.\mathrm{ID}_{\mathrm{D}} / \mathrm{IG}_{\mathrm{G}}\right)$ is also used to evaluate the changes in the atomic structure of graphene nanosheets [19]. The $\mathrm{ID}_{\mathrm{D}} / \mathrm{I}_{\mathrm{G}}$ for HGE and PP-MA/HGE masterbatch were measured to be 1.27 and 1.08, respectively, and the value of $\mathrm{I}_{\mathrm{D}} / \mathrm{I}_{\mathrm{G}}$ was further reduced after dynamic vulcanization (The $\mathrm{ID}_{\mathrm{D}} / \mathrm{I}_{\mathrm{G}}$ ratio of TPVs/HGE composite is 1.08). This may be due to the reduction of some remaining oxygen groups in the dynamic vulcanization process, which promotes the interfacial interaction between HGE and the TPVs matrix [20].

In conclusion, all the changes shown in the spectrum indicate that HGE and PP-MA form strong interfacial interaction.
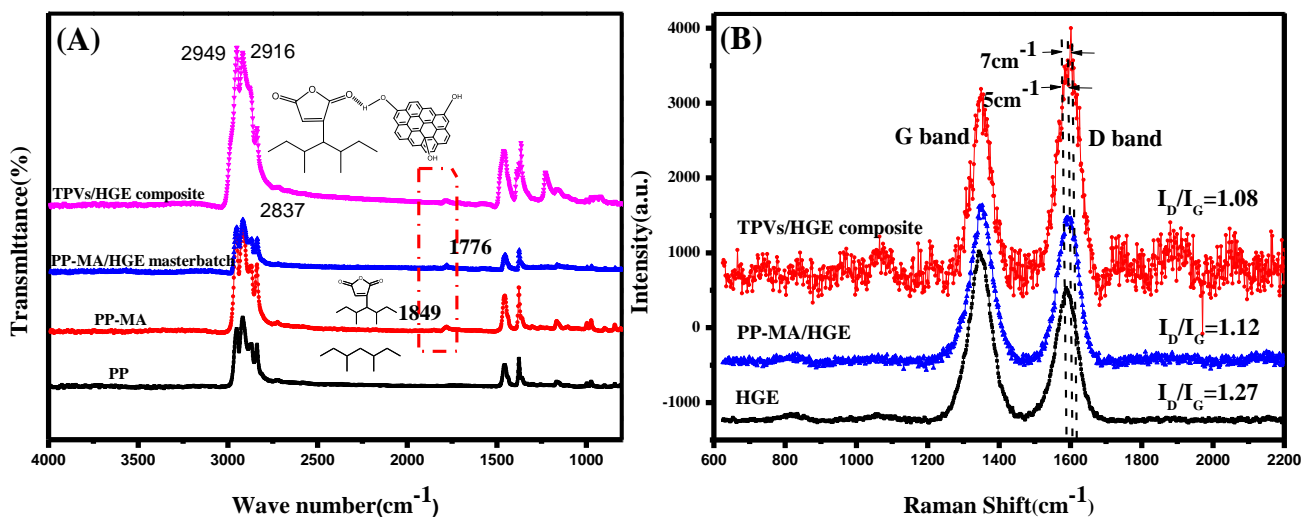

Figure 1. (A) FTIR spectra of PP, PP-MA, PP-MA/HGE masterbatch and TPVs/HGE composite and (B) Raman spectra of HGE, PP-MA/HGE masterbatch and TPVs/HGE composite

\subsection{Thermodynamic calculation of the wetting behavior of HGE in PP-MA and IIR}

From the analysis of the masterbatch, it can be known that there is a strong interfacial interaction between HGE and PP-MA. Since TPVs has special phase structure (high content of crosslinked rubber as the dispersed phase and low content of thermoplastic as the continuous phase), we hope to build nanosheet network by uniformly dispersing HGE in a low-content thermoplastic resin. However, in the process of melt blending, the dispersion of HGE in the two phases (PP-MA and IIR) has an important influence on the construction of the nanosheet network. We choose thermodynamic calculations to predict the dispersion of HGE in the two phases.

Wetting behavior of fillers at the two phases usually includes three cases, as shown in Figure 2. Wetting coefficient wa can be calculated by the following Equations [21]. 


$$
\omega \mathrm{a}=\frac{\gamma_{\mathrm{HGE}-\mathrm{IIR}} \pm \gamma_{\mathrm{HGE}-(\mathrm{PP}-\mathrm{MA})}}{\gamma_{\mathrm{IIR}-(\mathrm{PP}-\mathrm{MA})}}
$$

Where, $\gamma_{\mathrm{HGE}-\mathrm{IIR}}$ is the interfacial tension between HGE and IIR, $\gamma_{\mathrm{HGE}-(\mathrm{PP}-\mathrm{MA})}$ is the interfacial tension between HGE and PP-MA, and $\gamma_{\text {IIR-(PP-MA) }}$ is the interfacial tension between IIR and PP-MA.

(a) when wa $<-1$, the HGE will tend to go into the IIR phase, as shown in Figure 2(A).

(b) when $-1<\omega \mathrm{a}<1$, the HGE will tend to the interface between IIR and PP-MA, as shown in Figure 2(B). 2(C).

(c) when wa $>1$, the HGE will tend to go into the PP-MA phase, as shown in Figure

To calculate the interfacial tension between two components, the Harmonic-mean and Geometric-mean equations were used, as shown in Equations (2-3) [22].

Harmonic-mean equation:

$$
\gamma_{12}=\gamma_{1}+\gamma_{2}-4\left(\frac{\gamma_{1}^{d} \gamma_{2}^{d}}{\gamma_{1}^{d}+\gamma_{2}^{d}}+\frac{\gamma_{1}^{p} \gamma_{2}^{p}}{\gamma_{1}^{p}+\gamma_{2}^{p}}\right)
$$

And Geometric-mean equation:

$$
\gamma_{12}=\gamma_{1}+\gamma_{2}-2\left(\sqrt{\gamma_{1}^{d} \gamma_{2}^{d}}+\sqrt{\gamma_{1}^{p} \gamma_{2}^{p}}\right)
$$

Where $\gamma_{i}$ represents the total surface energy of the component $\mathrm{i}$, and $\gamma_{d}^{\mathrm{i}}$ and $\gamma_{p}^{\mathrm{i}}$ represent the dispersive and polar force of surface energy of component $i$, respectively.

The surface energy of different components is calculated using Geometric-mean method in Equations (4-5) [23].

$$
\begin{aligned}
& \gamma=\gamma^{d}+\gamma^{p} \\
& \gamma_{l}(1+\cos \theta)=2\left(\gamma_{s}^{d} \gamma_{l}^{d}\right)^{1 / 2}+2\left(\gamma_{s}^{p} \gamma_{l}^{p}\right)^{1 / 2}
\end{aligned}
$$

Where $\theta$ is the observed contact angle, $\gamma_{s}$ and $\gamma_{l}$ are the surface energy of the solid and liquid, respectively, $\gamma_{d}^{\mathrm{s}}$ and $\gamma_{p}^{\mathrm{s}}$ are the solid dispersion force and polar force, and $\gamma_{d}^{\mathrm{l}}$ and $\gamma_{p}^{\mathrm{l}}$ are the liquid dispersion force and polar force, respectively.

The contact angle of non-polar liquid $\mathrm{H}_{2} \mathrm{O}$ and polar liquid $\mathrm{C}_{3} \mathrm{H}_{8} \mathrm{O}_{3}$ on the surfaces of IIR the and PP-MA films were $63.4^{\circ}$ and $91.0^{\circ}, 88.6^{\circ}$ and $81.5^{\circ}$, respectively. To calculate the surface energy of IIR and PP-MA, one must know the corresponding parameters of the $\mathrm{H}_{2} \mathrm{O}$ and $\mathrm{C}_{3} \mathrm{H}_{8} \mathrm{O}_{3}$. The parameters of $\gamma^{p}=51.0 \mathrm{~mJ} / \mathrm{m}^{2}$ and $\gamma^{d}=21.8 \mathrm{~mJ} / \mathrm{m}^{2}$ for the $\mathrm{H}_{2} \mathrm{O}$, and $\gamma^{p}=22.8 \mathrm{~mJ} / \mathrm{m}^{2}$ and $\gamma^{d}=40.6 \mathrm{~mJ} / \mathrm{m}^{2}$ for the $\mathrm{C}_{3} \mathrm{H}_{8} \mathrm{O}_{3}$. The surface energy of graphene is reported by a highly cited paper to be $42.8 \mathrm{~mJ} / \mathrm{m}^{2}$, and the $\gamma^{p}$ and $\gamma^{d}$ are $2.4 \mathrm{~mJ} / \mathrm{m}^{2}$ and $40.4 \mathrm{~mJ} / \mathrm{m}^{2}$, respectively [24-25]. The surface energy data of IIR, PP-MA and HGE was shown in Table 1, The interface energy data of IIR/HGE, PP-MA/HGE and IIR/PP-MA was shown in Table 2.

The wa was calculated as 1.95 based on Harmonic-mean equation and 1.70 based on Geometric-mean equation. Obviously, the thermodynamic calculation indicates that HGE have a tendency to preferentially localize at the PP-MA phase during melt-blending processing. From the contact angle analysis, we know that HGE is mainly dispersed in PPMA. Therefore, if the content of HGE is large enough, HGE will overlap each other to form sheeted network. 

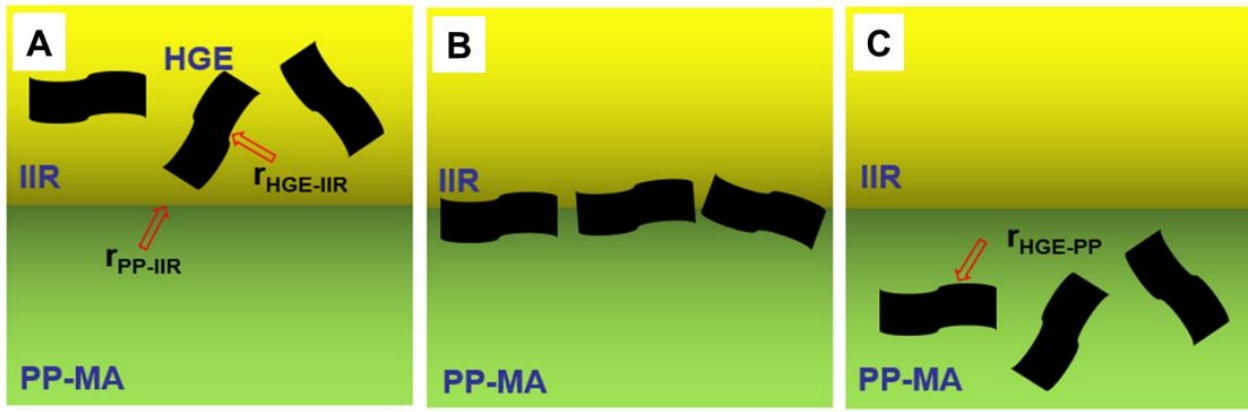

Figure 2. Wetting behavior of fillers in PP-MA and IIR

Table 1. Surface energy data for IIR, PP-MA and HGE.

\begin{tabular}{cccc}
\hline Component & $\gamma^{p}\left(\mathrm{~mJ} / \mathbf{m}^{2}\right)$ & $\gamma^{d}\left(\mathbf{m J} / \mathbf{m}^{2}\right)$ & $\gamma\left(\mathbf{m J} / \mathbf{m}^{2}\right)$ \\
\hline IIR & 37.05 & 0.14 & 37.19 \\
PP-MA & 1.11 & 41.41 & 42.52 \\
HGE & 2.40 & 40.40 & 42.80 \\
\hline
\end{tabular}

Table 2. Interfacial energy data of IIR/HGE, PP-MA/HGE and IIR/PP-MA.

\begin{tabular}{ccc}
\hline Samples & $\begin{array}{c}\text { Based on Harmonic-mean } \\
\text { equation }\left(\mathbf{m} \mathbf{J} / \mathbf{m}^{2}\right)\end{array}$ & $\begin{array}{c}\text { Based on Geometric-mean } \\
\text { equation }\left(\mathbf{m} / \mathbf{m}^{2}\right)\end{array}$ \\
\hline IIR/HGE & 70.40 & 56.40 \\
PP-MA/HGE & 0.48 & 0.25 \\
IIR/PP-MA & 74.84 & 32.90 \\
\hline
\end{tabular}

\subsection{The morphology of TPVs/HGE composites}

The main reasons why we can build the nanosheet network structure in TPVs through masterbatch technology are as follows: (1) The interfacial interaction between HGE and PP-MA was stronger than between IIR and HGE through PP-MA/HGE masterbatch analysis, (2) It was very difficult for HGE to migrate from masterbatch to IIR rubber during melting blending process or dynamic vulcanization by thermodynamic calculation.

To show more clearly the network of HGE in the TPVs/HGE composites, the morphology of composites was characterized by using TEM and the results were shown in Figure 3. Since the sample was stained with osmium tetroxide, the continuous whiter regions were PP or PP-MA matrix, the dispersed darker particles were IIR rubber, and the nanosheets were HGE. It can be seen from Figure 3 that most HGE sheets were dispersed in the PP (PP-MA) matrix with a crumple and wrinkle structure. When the HGE content in the composites was less than $2 \%$ (see Figure 3(B)), the HGE could uniformly dispersed in the matrix without interconnection. HGE started to interconnect with one another in the composites with $2.0 \mathrm{wt} . \%$ of HGE (see Figure 3(C, D)), which indicates that the nanosheet network of HGE was formed in the TPV matrix. On the other hand, we can also see that rubber particles occupies most of the volume of the PP matrix in the composites, which would hinder the aggregation of HGE and promote the formation of an effective nanosheet network. Thus, the rubber particles and HGE together form the nanosheet network in TPVs/HGE composites.

The introduction of HGE will also improve the interfacial bonding of the rubber and plastic phases in the TPVs composite matrix, which will be explained in detail in the next section. 


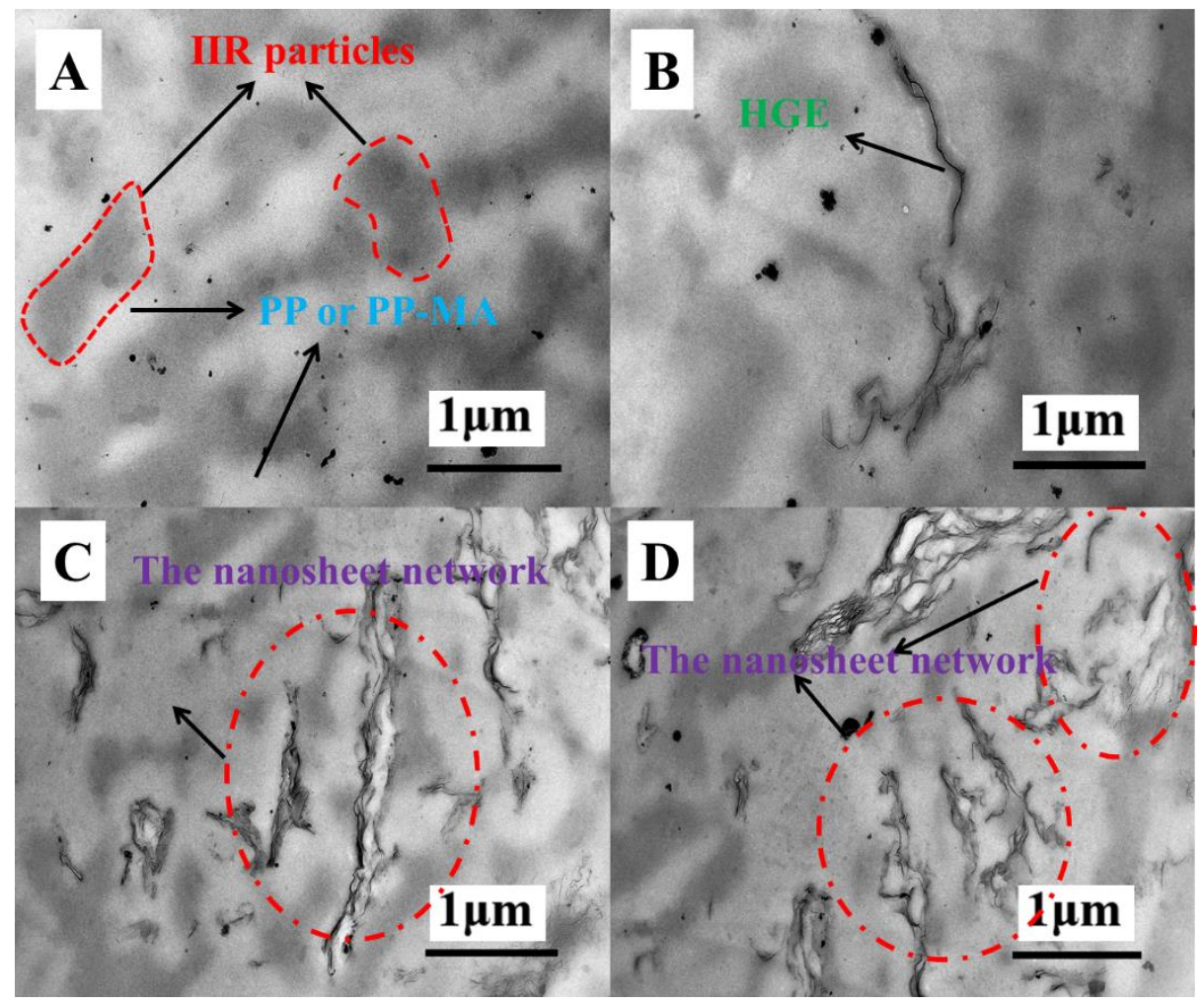

Figure 3. TEM images of pure TPVs and TPVs/HGE composites with different contents of HGE: (A) pure TPVs, (B) TPVs/HGE 0.5 wt.\% composites, (C) TPVs/HGE 2 wt.\% composites, (D) TPVs/HGE 3 wt.\% composites.

\subsection{Mechanical properties}

The mechanical properties of pure TPVs and TPVs/HGE composites with different contents of HGE were measured, and the results were shown in Table 3. The tensile strength of TPVs/HGE composites increased with the increase in HGE concentration, and the respective improvements were $\sim 13.5 \%$ and $\sim 24.6 \%$ for the HGE at the loading of 0.5 wt.\% and 1 wt.\% HGE. It can be seen from Table 3 that the maximal reinforcements in tensile strength was $34.6 \%$ achieved at 3 wt.\% HGE loading, then there was a downturn when the content of HGE increase to $5 \mathrm{wt} . \%$. The reason for the decrease in the mechanical properties of composites is that it is difficult to completely disperse the nanofillers through melt processing at high loading, and many defects will be introduced into the matrix. However, the elongation at break decreased with increasing HGE concentration. Similar phenomena were widely reported about nanofillers reinforced composites [26-27]. The hardness of TPVs/HGE composites did not change significantly with loading. The hardness of nanocomposites seems to mainly depend on the content of particles. When the content of particles is low, the hardness does not change significantly.

Compared with pure TPVs, the tensile strength of the TPVs/HGE $2 \mathrm{wt} . \%$ composites increased from 10.3 MPa to $13.8 \mathrm{MPa}$ (increased by about 31.6\%), which indicates that the nanosheet network can significantly improve the mechanical properties. To explore the interface bonding between PP and IIR, the tensile fracture surfaces of pure TPVs and TPVs/HGE composites (at 2 wt.\% HGE) were observed by using SEM (see Figure 4(A, C)). It can be observed that many rubber particles of pure TPVs were pulled out to form filamentous domains, which indicates that the rubber and plastic phases in the TPVs matrix are severely separated. However, the tensile fracture surfaces of the TPVs/HGE composites shown relatively rough, and no obvious IIR particles were pulled out, which indicates that the nanosheet network could improve the interface interaction between IIR and PP. 
In order to clearly observe the interface bonding between HGE and TPVs matrix, we used SEM image with high magnification (see Figure 4(D)). It can be seen from the highresolution SEM photographs that the TPVs matrix is attached to the surface of the HGE, indicating that the interface structure between HGE and TPVs was firm, A Possible mechanism for HGE grafted to IIR network in the dynamic vulcanization process was shown in Figure 5. The hydroxylated HGE can be combined with the IIR cross-linked network during dynamic vulcanization, and enhances the interface strength of HGE and TPVs matrix, which was consistent with the improvement of tensile strength.

Table 3. Tensile data of TPVs/HGE composites with different HGE contents.

\begin{tabular}{cccc}
\hline Sample & Shore A & $\begin{array}{c}\text { Tensile } \\
\text { strength } \\
\text { (MPa) }\end{array}$ & $\begin{array}{c}\text { Elongation at } \\
\text { break (\%) }\end{array}$ \\
\hline Neat TPVs & 80 & 10.36 & 296 \\
TPVs/HGE 0.5 wt.\% & 80 & 11.76 & 276 \\
TPVs/HGE 1 wt.\% & 81 & 12.91 & 246 \\
TPVs/HGE 2 wt.\% & 82 & 13.64 & 212 \\
TPVs/HGE 3 wt.\% & 83 & 13.94 & 203 \\
TPVs/HGE 5 wt.\% & 83 & 12.96 & 189 \\
\hline
\end{tabular}

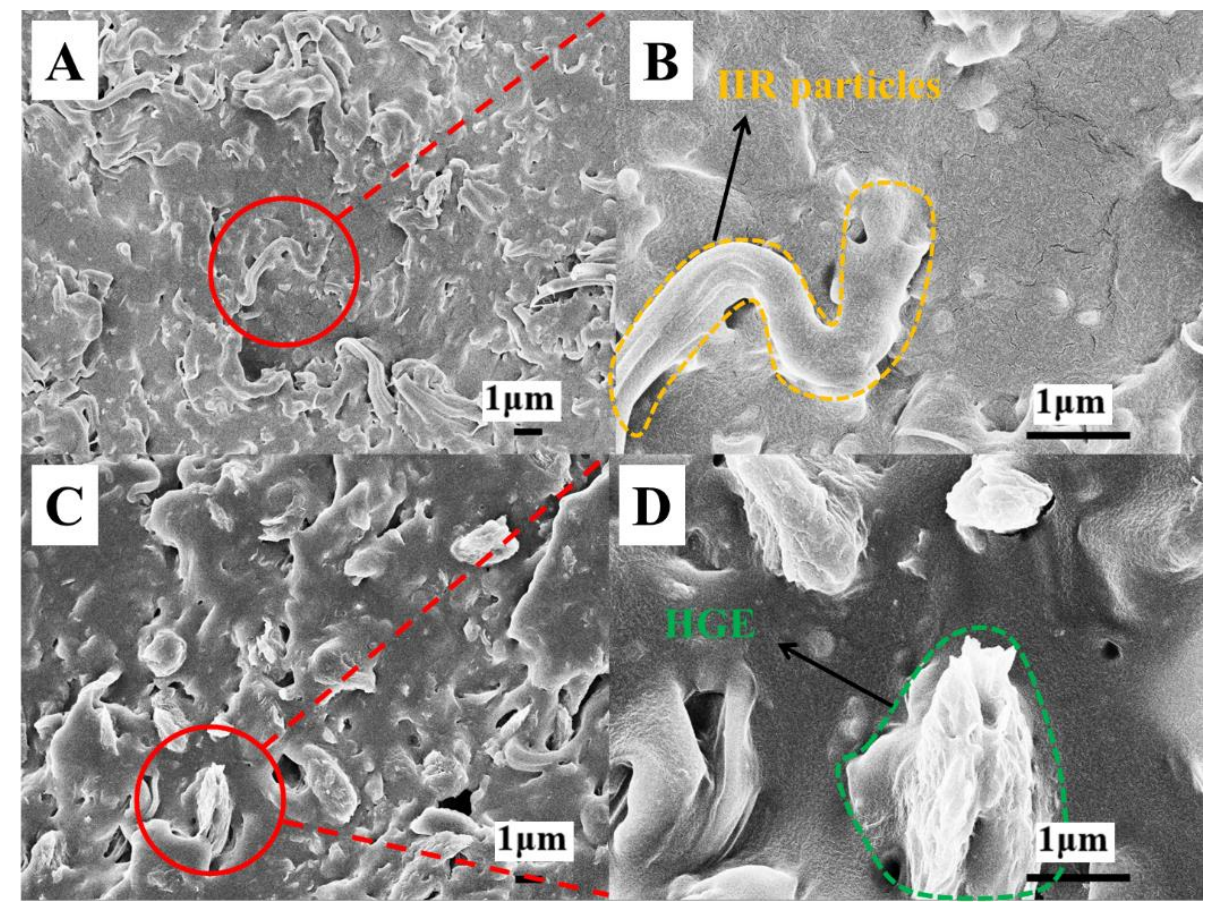

Figure 4. SEM micrographs of the tensile fracture surfaces ( $B$ and $D$ are the magnified images of $A$ and $C$, respectively): (A) pure TPVs, (B) TPVs/HGE 2 wt.\% composites

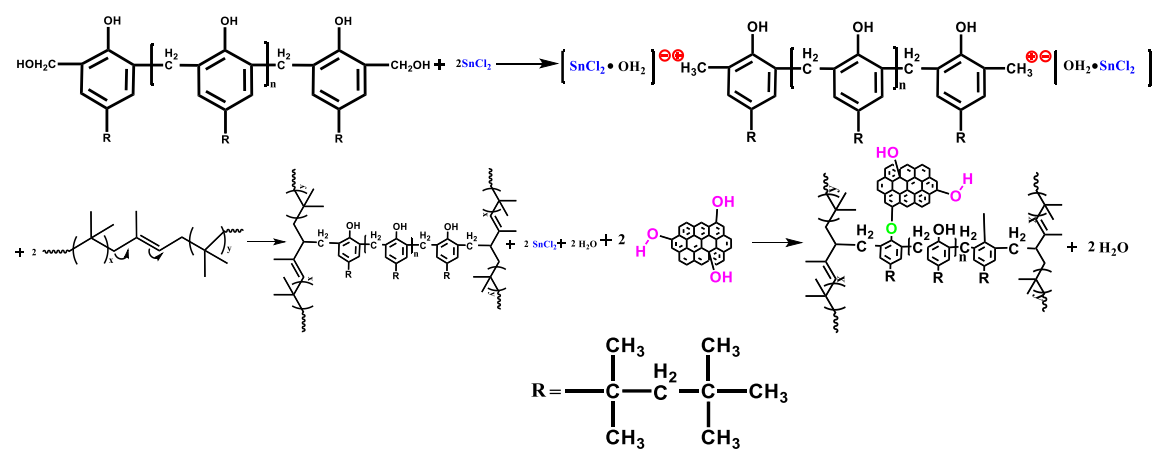


Figure 5. A Possible mechanism for HGE grafted to IIR network in the dynamic vulcanization of IIR/PP TPVS

\subsection{Electrical properties}

To study the effect of the nanosheet network on the electrical properties of TPVs/HGE composites, we measured the dielectric properties of the pure TPVs and all the TPVs/HGE composites in the frequency range of $10^{0}-10^{6} \mathrm{~Hz}$ and the results were shown in Figure 6. In order to more clearly show the influence of the nanosheet network on the composites, Figure 6(B) shows the variation of AC conductivity with different HGE weight fraction at $10^{2} \mathrm{~Hz}$. It can be seen from the Figure 6(B) that as the incorporation of HGE increased, the AC conductivity of TPVs/HGE composites increased sharply at a critical concentration (at $2 \mathrm{wt} . \%$ ) of HGE, indicating that the nanosheet network was formed. Therefore, we define a content of HGE of $2 \mathrm{wt} . \%$ as the threshold percolation of the TPVs/HGE composites [2829]. The nanosheet network could improve the electrical properties of TPVs/HGE composites including several factors: (1) HGE exhibit unique atomic structure and large surface area, (2) HGE could be evenly dispersed in the PP (PP-MA) matrix via the masterbatch technique, (3) large numbers of rubber particles are dispersed in the PP (PP-MA) matrix to hinder the of aggregation of HGE and improve the formation of conductive paths.

Figure $6(\mathrm{C})$ presents the frequency dependence of the dielectric permittivity of TPVs/HGE composites with different HGE contents. With the increase of HGE amount, the dielectric permittivity increased significantly, especially when the HGE loading reached $2 \mathrm{wt} . \%$. This again indicates that a content of HGE of $2 \mathrm{wt} . \%$ is the percolation threshold of the composites. The high dielectric permittivity was mainly attributed to the interface polarization effect between the HGE and TPVs matrix. The principle of MaxwellWagner polarization can further explain this phenomenon, and charge-dipoles due to good dispersion of HGE in the PP (PP-MA) matrix and IIR domain [30]. On the other hand, the filler-filler distance became smaller improved the nanosheet network and further promotes the interface polarization. The decrease of dielectric permittivity with frequency might be due to lesser time available for dielectric relaxation or dipole orientation during each cycle [31].

The change trend of the loss tangent of TPVs/HGE composites with frequency was consistent with the dielectric permittivity, as shown in the Figure 6(D). For example, the loss tangent of the TPVs/HGE $1 \mathrm{wt} . \%$ composites was 0.51 (dielectric permittivity 3.35), while the loss tangent of the TPVs/HGE 2 wt.\% composites increases to about 0.72 (dielectric permittivity 5.38). For nanocomposites containing conductive nanofillers, the main reason for the dielectric loss should be the leakage current existed in the nanocomposites, especially when the content of nanofillers is high, which seems to be an inherent feature of this material.
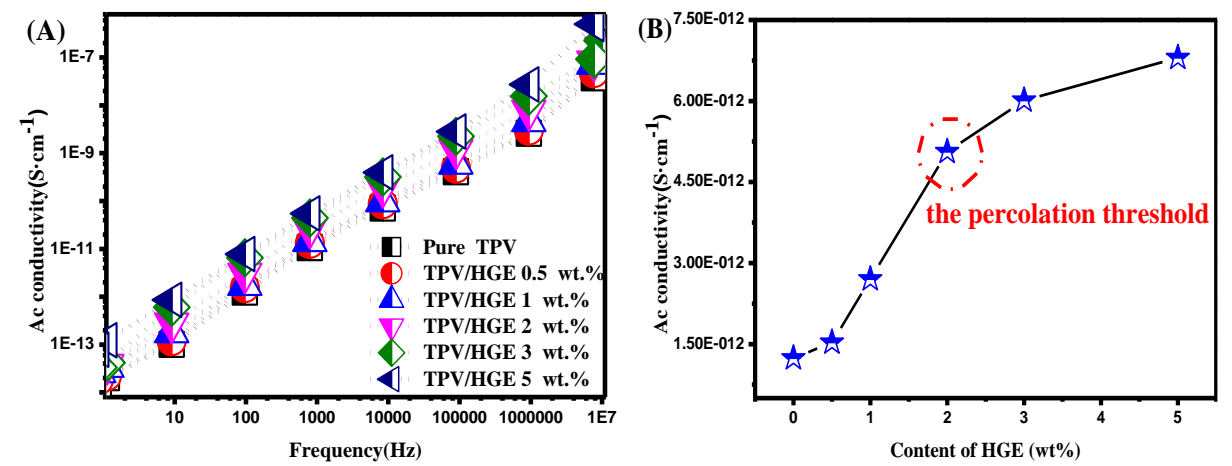

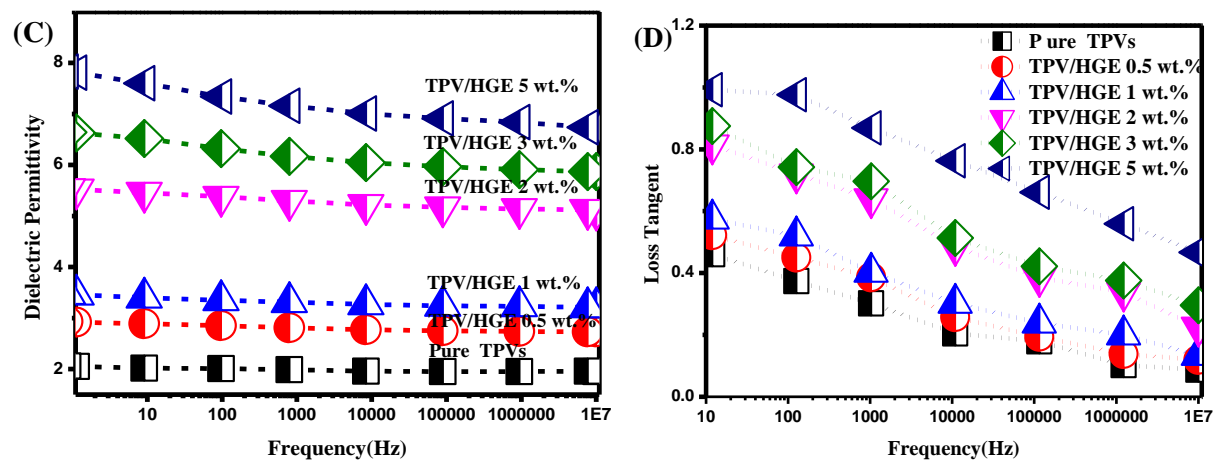

Figure 6. Effect of different HGE contents on dielectric properties of TPVs/HGE composites: (A) AC conductivity vs. frequency, (B) AC conductivity of TPVs/HGE composites with different HGE contents at $10^{2} \mathrm{~Hz},(\mathrm{C})$ Dielectric constant vs. frequency, (D) loss tangent vs. frequency.

\subsection{Thermal properties}

To study the effect of the nanosheet network on the thermal properties of TPVs/HGE composites, we measured the thermal conductivity of pristine PP-MA, IIR, TPVs and their composites based on HGE as nanofillers, and the results were shown in Figure 7(A). The weight fraction of HGE in all polymers is $2 \mathrm{wt} . \%$. We can see from the picture that the thermal conductivity of PP-MA/HGE masterbatch was increased by $29.4 \%$ compared to pure PP-MA, which indicate that HGE would eventually improve the intrinsic thermal conductivity of PP-MA through the masterbatch technique. The increase in thermal conductivity of IIR/HGE composites compared to pure IIR (8.1\%) is not obvious, mainly due to IIR is non-polar, HGE is unevenly dispersed in IIR vulcanizate, HGE network was weak, and heat transfer efficiency was low. Although the thermal conductivity of IIR is lower than that of PP-MA, the cross-linked rubber particles can hinder the aggregation of HGE and promote the formation of the nanosheet network. Therefore, thermal conductivity of the TPVs/HGE composite was higher than that of neat TPVs (increased 25.9\%), mainly because the unique nanosheet network improves the thermal conductivity.

Figure $7(\mathrm{~B})$ shown the room-temperature thermal conductive parameters of the TPVs/HGE composites as a function of HGE content. As expected, the thermal conductivity of the composites increases sharply as the content of HGE increases. The thermal conductivity of pure TPVs is $0.116 \mathrm{~W} \cdot \mathrm{mk}^{-1}$ when the HGE are incorporated and the loadings of HGE are $1 \%$ and $3 \%$, the thermal conductivity reached $0.131 \mathrm{~W} \cdot \mathrm{mk}^{-1}$ and $0.152 \mathrm{~W} \cdot \mathrm{mk}^{-1}$, which correspond to the increase by $13 \%$ and $31 \%$, respectively. About the percolation threshold, the thermal conductivity reached a plateau and the value could achieve to 0.160 $\mathrm{W} \cdot \mathrm{mk}^{-1}$ when the HGE loading was $2 \mathrm{wt} . \%$. The HGE uniformly dispersed in the PP matrix of the TPVs/HGE composites could overlaps each other to form an effective thermally conductive nanosheet network, which reduces thermal resistance and facilitates heat transfer.
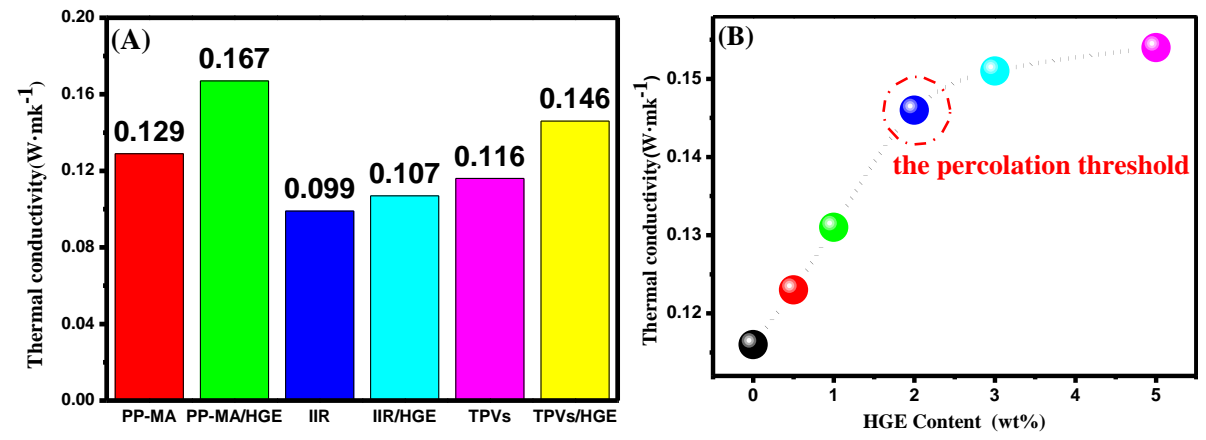

Figure 7. (A) Thermal conductivity of pristine PP-MA, IIR, TPVs and their composites based on HGE as nanofillers, (B) Thermal conductivity of TPVs/HGE composites with various contents of HGE. 


\subsection{The nanosheet network in TPVs/HGE composites}

Based on the above results, we propose a schematic representation of the nanosheet network in TPVs/HGE composites, as shown in Figure 8. The rubber particles in the TPVs/HGE composites occupies most of the volume of the PP phase, which would hinder the aggregation of HGE and form the effective nanosheet network. PP-MA as compatibilizer can not only significantly improve the interfacial interaction between HGE and PP, but also promote the uniform dispersion of HGE. When the HGE reaches the percolation threshold ( $2 \mathrm{wt} . \%)$, the HGE in the PP phase gradually overlap with one another and form nanosheet network in the matrix, as shown in the Figure 8(B). Thus, the thermoelectric and mechanical properties of TPVs increased sharply, and we define this network as the nanosheet network of the composites. The nanosheet network in the composite after percolation threshold can improve the dielectric properties, thermal properties and mechanical properties.

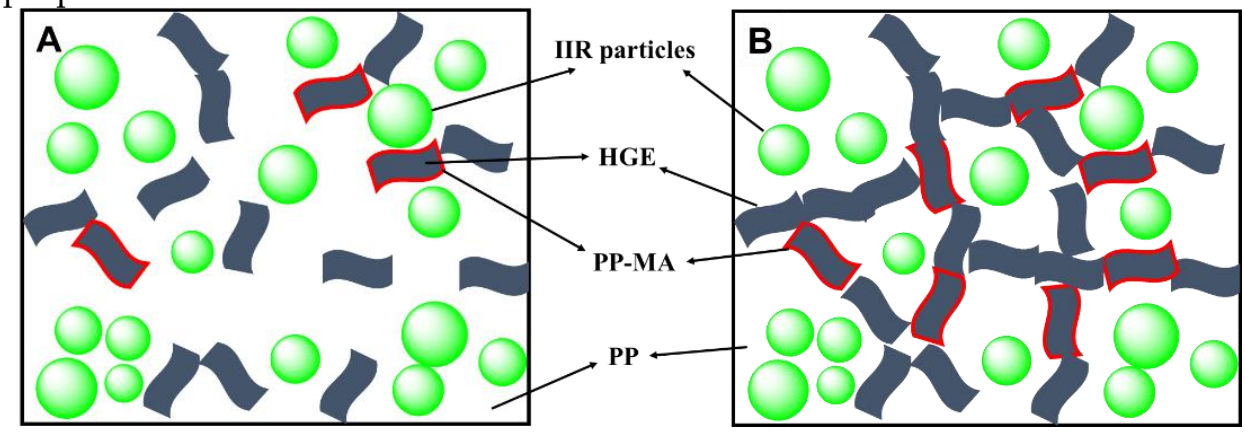

Figure 8. Schematic of the nanosheet network in TPVs/HGE composites:(A) before percolation threshold, (B) after percolation threshold

\section{Conclusions}

In this work, the TPVs/HGE composites with nanosheet network were prepared through masterbatch technique strategy and based on thermodynamic calculations, using PP-MA as a compatibilizer. The strong interfacial interaction between HGE and the compatibilizer was shown by FTIR and Raman spectra. The nanosheet network was formed by HGE interconnect with one another. The rubber particles occupied most of the volume the PP phase, hindering the aggregation of HGE and promoting the formation of the nano network. The nanosheet network improved the mechanical strength of TPVs mainly because HGE could combine with the IIR cross-linked network during the dynamic vulcanization process to improve the interface bonding strength between PP and IIR. When the HGE content reached the percolation threshold (2 wt.\%), the nanosheet network of HGE was formed, the AC conductivity, dielectric permittivity and thermal conductivity increased sharply. As a result, the prepared TPVs/HGE nanocomposites simultaneously possessed higher mechanical properties, thermal properties and dielectric properties. This work provides a guideline on the preparation of high-performance conductive non-polar TPVs for practical applications.

Author Contributions: Conceptualization, Q.T. and Y.Z.; methodology, X.L.; software, L.C.; writing - review and editing, Q.T.; funding acquisition, Z.Z. All authors have read and agreed to the published version of the manuscript.

Funding: This research was funded by Natural Science Foundation of Shandong Province, grant number ZR201911070097

Conflicts of Interest: The authors declare no conflict of interest.

\section{References}

1. Ning, N.Y.; Li, S.Q.; Wu, H.G.; Tian, H.C.; Yao, P.J.; Hu, G.H.; Tian, M.; Zhang, L.Q. Preparation, microstructure, and microstructure-properties relationship of thermoplastic vulcanizates (TPVs): A review. Prog.Polym.Sci. 2018, 79, 61-97. 
2. Wu, D.F.; Lv, Q.L.; Feng, S.H.; Chen, J.X.; Chen, Y.; Qiu, Y.X.; Yao, X. Polylactide composite foams containing carbon nanotubes and carbon black: Synergistic effect of filler on electrical conductivity. Carbon 2015, 95, 380-387.

3. Li, Q.; Xue, Q.; Zheng, Q.; Hao, L.Z.; Gao, X.L. Large dielectric constant of the chemically purified carbon nanotube/polymer composites. Materials Letters 2008, 62, 4229-4231.

4. Yoshihiko, K.; Yoshikawa, H.; Kunio, A.; Masaki, M.; Tatsuo, T.; Kayano, S.; Bandow, S.J.; Sumio, L. Preparation, photocatalytic activities, and dye-sensitized solar-cell performance of submicron-scale TiO2 hollow spheres. Langmuir 2008, 24, 547-550.

5. Yang, D.; Zhang, L. Q.; Liu, H. L.; Dong, Y.C. Lead magnesium niobate-filled silicone dielectric elastomer with large actuated strain. Appl. Polym 2012, 125, 2196-2201.

6. Tian, H.C.; Tian, M.; Zou, H.; Dang, Z.M. Special electrical conductivity of carbon black-filled two-phased thermoplastic vulcanizates. Journal of Applied Polymer Science 2010, 117, 691-699.

7. Yan, N.; Xia, H.S.; Wu, J.K.; Zhan, G.X.; Chen, C. Compatibilization of natural rubber/high density polyethylene thermoplastic vulcanizate with graphene oxide through ultrasonically assisted latex mixing. Journal of Applied Polymer Science 2012, 127, 933941.

8. Li, C. Q.; Zha, J.W.; Li, Z.J.; Zhang, D.L.; Wang, S.J.; Dang, Z.M. Towards balanced mechanical and electrical properties of thermoplastic vulcanizates composites via unique synergistic effects of single-walled carbon nanotubes and graphene. Composites Science and Technology 2018, 157, 134-143.

9. Novoselov, K. S.; Geim, A. K.; Morozov, S. V.; Jiang, D.; Katsnelson, M.L.; Grigorieva, I.V.; Dubonos, S.V.; Firsov, A.A. Nature 2005, 438, 197-200.

10. Kim, H.; Abdala, A.A.; Macosko, C.W. Graphene/Polymer Nanocomposites. Macromolecules 2010, 43, 6515-6530.

11. Weiss, N. O.; Zhou, H.L.; Liao, L.; Liu, L.; Jiang, S.; Huang, Y.; Duan, X.F. Graphene: an emerging electronic material. Advanced Materials 2012, 24, 782-825.

12. Liang, J. Z.; Du, Q.; Tsui, G. C.P.; Tang, C. Y. Tensile properties of graphene nano-platelets reinforced polypropylene composites. Composites Part B: Engineering 2016, 95, 166-171.

13. Sethuraman, K.; Alagar, M. Thermo-mechanical and dielectric properties of graphene reinforced caprolactam cardanol based benzoxazine-epoxy nanocomposites. Rsc Advances 2015, 5, 9607-9617.

14. Yang, B. X.; Shi, J. H.; Pramoda, K. P.; Goh, S.H. Enhancement of the mechanical properties of polypropylene using polypropylene-grafted multiwalled carbon nanotubes. Composites Science and Technology 2008, 68, 2490-2497.

15. Madni, I.; Hwang, C. Y.; Park, S. D.; Choa, Y.H.; Kim, H.T. Mixed surfactant system for stable suspension of multiwalled carbon nanotubes. Colloids \& Surfaces A Physicochemical \& Engineering Aspects 2010, 358, 101-107.

16. Yuan, B.H.; Bao, C.L.; Song, L.; Hong, N.N.; Liew, K.M.; Hu, Y. Preparation of functionalized graphene oxide/polypropylene nanocomposite with significantly improved thermal stability and studies on the crystallization behavior and mechanical properties. Chemical Engineering Journal 2014, 237, 411-420.

17. Wang, D.R.; Zhang, X.M.; Zha, J. W.; Zhao, J.; Dang, Z.M.; Hu, G.H. Dielectric properties of reduced graphene oxide/polypropylene composites with ultralow percolation threshold. Polymer 2013, 54, 1916-1922.

18. Li, C. Q.; Zha, J. W.; Long, H. Q.; Wang, S.J.; Zhang, D.L.; Dang, Z.M. Mechanical and dielectric properties of graphene incorporated polypropylene nanocomposites using polypropylene-graft-maleic anhydride as a compatibilizer. Composites Science and Technology 2017, 153, 111-118.

19. Song, P.G.; Cao, Z.H.; Cai, Y.Z.; Zhao, L.P.; Fang, Z.P.; Fu, S.Y. Fabrication of exfoliated graphene-based polypropylene nanocomposites with enhanced mechanical and thermal properties. Polymer 2011, 52, 4001-4010.

20. Mohiuddin, T. M. G.; Lombardo, A.; Nair, R. R.; Bonetti, A.; Savini, G.; Jalil, R.; Bonini, N.; Basko, D.M.; Galiotis, C.; Marzari, N. Uniaxial strain in graphene by Raman spectroscopy: G peak splitting, Grueneisen parameters, and sample orientation. Physical review 2009, 79, 205433-205433

21. Sumita, M.; Sakata, K.; Asai, S.; Miyasaka, K.; Nakagawa, H. Dispersion of fillers and the electrical conductivity of polymer blends filled with carbon black. Polymer Bulletin 1991, 25, 265-271.

22. Wu, S.H. Polymer interface and adhesion, 1rd ed.; Marcel Dekker Inc: Boca Raton, New York, 1982; pp. $256-278$.

23. Owens, D. K.; Wendt, R. C. Estimation of the surface free energy of polymers. Journal of Applied Polymer Science 1969, 13, 17411747.

24. Perrozzi, F.; Croce, S.; Treossi, E.; Palermo, V.; Santucci, S.; Fioravanti, G.; Ottaviano, L. Reduction dependent wetting properties of graphene oxide. Carbon 2014, 77, 473-480.

25. Wang, S.; Zhang, Y.; Abidi, N.; Cabrales, L. Wettability and Surface Free Energy of Graphene Films. Langmuir 2009, 25, 1107811081.

26. Huy, T. A.; Luepke, T.; Radusch, H. J. Characterization of the deformation behavior of dynamic vulcanizates by FTIR spectroscopy. Journal of Applied Polymer Science 2001, 80, 148-158.

27. Koval'chuk, A. A.; Shevchenko, V. G.; Shchegolikhin, A.; Nedorezova, P. M. Effect of Carbon Nanotube Functionalization on the Structural and Mechanical Properties of Polypropylene/MWCNT Composites. Macromolecules 2008, 41, 7536-7542.

28. Dou, R.; Shao, Y.; Li, S. L.; Yin, B.; Yang, M.B. Structuring tri-continuous structure multiphase composites with ultralow conductive percolation threshold and excellent electromagnetic shielding effectiveness using simple melt mixing. Polymer 2016, 83, 34-39. 
29. Zhu, Y.L.; Zhang, X.H.; Song, Z.H.; Qi, G.C. The effect of selective location of carbon nanotubes on electrical properties of thermoplastic vulcanizates. Journal of Applied Polymer Science 2013, 5, 3885-3890.

30. Xia, X. D.; Zhong, Z.; Weng, G. J. Maxwell-Wagner-Sillars mechanism in the frequency dependence of electrical conductivity and dielectric permittivity of graphene-polymer nanocomposites. Mechanics of Materials 2017, 109, 42-52.

31. Salaeh S. Processing of natural rubber composites and blends: relation between structure and properties. Ph.D. Thesis, University Claude Bernard-Lyon I, France, 2014. 